\title{
Gastric Mucormycosis by Rhizopus microsporus in a Patient with Uncontrolled Diabetes Mellitus
}

\section{Gitali Bhagawati ${ }^{13 *}$, Mahesh Gupta ${ }^{2}$, Sania Paul ${ }^{1}$, Rekha Saji Kumar ${ }^{1}$ and Lincy ${ }^{3}$}

${ }^{1}$ Department of Microbiology, Dharamshila Narayana Superspeciality Hospital and Research Centre, New Delhi, India

${ }^{2}$ Department of Gastroenterology, Dharamshila Narayana Superspeciality Hospital and Research Centre, New Delhi, India

${ }^{3}$ Department of Infection Control, Dharamshila Narayana Superspeciality Hospital and Research Centre, New Delhi, India

*Corresponding Author: Gitali Bhagawati, Department of Microbiology, Dharamshila Narayana Superspeciality Hospital and Research Centre, New Delhi, India.
Received: April 26, 2021

Published: May 17, 2021

(C) All rights are reserved by Gitali

Bhagawati., et al.

\begin{abstract}
Mucormycosis is a rapidly progressive life-threatening angio-invasive fungal infection caused by ubiquitous filamentous fungi of the order Mucorales and class Zygomycetes. The disease spectrum ranges from involvement of sinuses, lung, brain and skin to disseminated fatal infections, especially in immunocompromised hosts. Cases of primary gastric mucormycosis are rare in literature. It can be lethal due to invasion of gastric mucosa by Mucorales. High index of clinical suspicion in patient coming with haematemesis and melena with immunocompromised status can help in early intervention. Common risk factor for gastrointestinal mucormycosis (GIM) has been found to be uncontrolled DM with and without diabetic ketoacidosis (DKA). Here, we are presenting a case of 48 year male patient diagnosed with mucormycosis of gastric fundus coming with history of melena of one week duration and single episode of haematemesis. The patient had uncontrolled DM and was on oral hypoglycemic agent. Fungal culture showed growth of Mucor. Morphology of the fungal colonies as well as microscopic examination of the fungus confirmed it as Rhizopus spp. Fungal colonies were sent for further confirmation by molecular sequencing to reference laboratory. Species level identification was done by ITS15.8S-ITS2 rRNA gene cluster internal transcribed spacer [ITS] sequencing. Sequencing confirmed the fungus as Rhizopus microsporus. The patient was put on intravenous Amphotericin-B; but was stopped in view of renal function derangement. Later Posaconazole was added and patient responded completely.
\end{abstract}

Keywords: Zygomycetes; Rhizopus microsporus; Gastrointestinal Mucormycosis; Immunocompromised

\section{Introduction}

Mucormycosis is a life- threatening infection caused by fungi belonging to the order Mucorales, in the subphylum Mucormycotina, class Zygomycetes [1,2]. These species are vasotropic, caus- ing rapid onset of tissue infarctions and necrosis and subsequent thrombosis by invading vascular bed [2,3]. There are six most commonly reported forms of mucormycosis which include rhinocerebral, pulmonary, cutaneous, gastrointestinal, disseminated, 
and uncommon presentations [4,5]. Out of these, gastrointestinal mucormycosis (GIM) is the most difficult to diagnose. Common risk factors for GIM include, low birth weight infants, organ transplants, haematological malignancies, neutropenia, diabetes mellitus (DM), chronic alcoholism, malnutrition, peritoneal dialysis, persistent ingestion of non-nutritional substances (pica), gastric ulcers, use of broad spectrum antibiotics and Covid-19 infection [3,6-8]. There is one reported outbreak of an iatrogenic gastric mucormycosis occurred due to contamination of wooden applicators that were used to mix drugs for patients with nasogastric feeding tubes [9].

The common sites of GIM include large intestine, stomach, small intestine and oesophagus $[3,10,11]$.

Disease outcome and mortality varies with the site of mucormycosis, however, it is very high with GIM (85\%) [12].

\section{Case History}

A 48 year old gentleman with blood group $A B+$ ve presented with melena of 6 days duration along with one episode of haematemesis 6 days back along with fever, pain on left lower leg and weight loss. He complained of marked generalized weakness and decreased appetite. There was no history of abdominal pain or distension.

His medical history consisted of uncontrolled Type-2 diabetes mellitus (DM) with body mass index (BMI) $21.5 \mathrm{~kg} / \mathrm{mt}^{2}$ and on oral hypoglyceamic agent (OHA) for last 5 years. He had no history of hypertension.

On examination, he was febrile $\left(100^{\circ} \mathrm{F}\right)$ with pulse rate $100 /$ min and normal blood pressure. He had joint stiffness and dizziness. There was no pallor, edema or abdominal pain at the time of admission. Routine blood investigation showed low Haemoglobin $(\mathrm{Hb})(8.22 \mathrm{mg} / \mathrm{dl})$ for which he was given one unit of packed red blood cells (PRBC) on Day 1. Renal function abnormality was seen with serum urea $42.8 \mathrm{mg} / \mathrm{dl}$ and serum creatinine $1.48 \mathrm{mg} /$ dl. Total leucocyte count was $11000 / \mu \mathrm{L}$. In view of hyperglycemia, Human insulin regular was started according to blood sugar before meals. Patient was put on injection Ceftriaxone and Metronidazole as empiric antibiotics. Patient was kept Nil per os (NPO) and Ryle's tube feeding was started.

On second day, upper gastro-intestinal endoscopy (UGIE) was done which showed large deep ulcerated area with perforated gas- tric wall in fundus. Ulcer base and edges were covered with clots, slough and necrotic tissue (Figure 1).
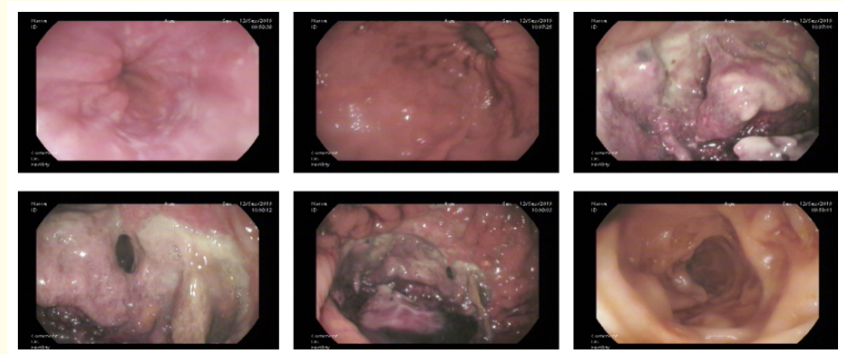

Figure 1: Large deep ulcerated area with perforated fundal wall with clot, slough, and necrotic tissue at the base of the ulcer and at edge. Active bleeding was seen at the base and margin of the ulcer. Esophagus, part of stomach (body, antrum and pylorus) and duodenal bulb was normal.

Biopsy was taken from margins for histopathological examination (HPE) which showed gastric mucormycosis. Contrast Enhanced Computed Tomography (CECT) abdomen showed irregular enhancing thickening in the gastric fundus with postero-superior discontinuity in the gastric wall. Resultant localized air-fluid contacting (dumb bell shaped) collection noted in the left subdiaphragmatic location with small intra-thoracic component and splenic sub-capsular component. Size of this collection was $6.5 \mathrm{~cm}$ (craniocaudal) x $3.6 \mathrm{~cm}$ (AP) x $6 \mathrm{~cm}$ (transverse). Gastric pyloroantral canal shows diffuse edematous rugal thickening. Mild left pleural effusion with basal atelectasis was seen. Bilateral lower lobes showed fissural based necrotic sub-centimeteric nodules. One PRBC was given on Day 2.

On Day 3, due to pain on left leg, venous/arterial doppler study was done which showed left lower limbs deep vein thrombosis (DVT).

On Day 3, peritoneal fluid was sent for fungal stain and fungal culture and sensitivity. $10 \% \mathrm{KOH}$ mount of the fluid showed hyaline non-septate, broad, ribbon like hyphae along with budding yeast cells (BYC). Sample was inoculated into two Sabouraud's Dextrose Agar (SDA) slants supplemented with chloramphenicol and kept them at $25^{\circ} \mathrm{C}$ and $37^{\circ} \mathrm{C}$. After 72 hours of incubation, two types of colonies observed. Both the colonies were sub-cultured on separate SDA tubes and re-incubated at $25^{\circ} \mathrm{C}$ and $37^{\circ} \mathrm{C}$. 
One type of colony was smooth, cream-colored, pasty white in colour. Gram stain and Lactophenol Cotton Blue (LPCB) showed small budding yeast cells without pseudohyphae. Further identification and antifungal susceptibility test of the yeast was done by YST Identification cards and YST-AST cards respectively using Vitek 2 Compact System (bioMérieux, Inc. Durham, North Carolina/USA). The identified isolate was Candida glabrata sensitive to all antifungals except caspofungin which showed intermediate sensitivity.

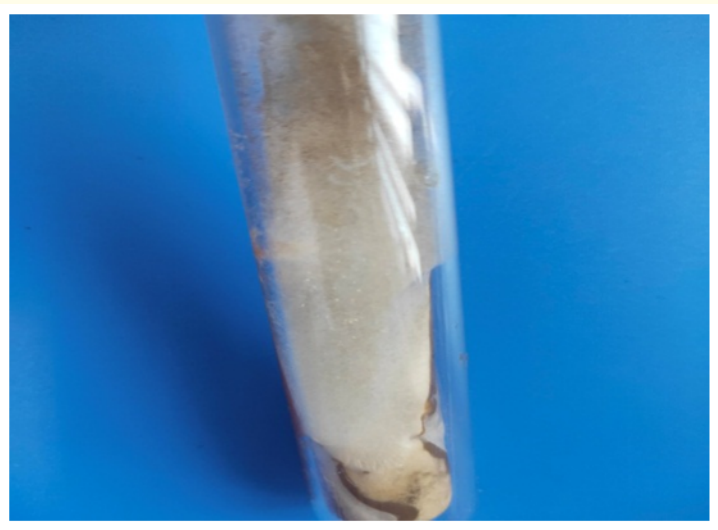

Figure 2: Fluffy, cotton candy like colony of Rhizopus spp. on Sabouraud's Dextrose Agar.

The other fungal growth was white fluffy, cotton-candy like growth in SDA with reverse pale yellow in colour (Figure 2). Slide culture was done. LPCB mount showed short to medium sporangiophores, pyriform to elliptical columella, subglobose and slightly angular sporangiospores were seen (Figure 3). Looking at the colony morphology and microscopic structures, the fungus was identified as Rhizopus spp.

For further species level identification, growth was sent for molecular identification to referral laboratory. Internal Transcribed Spacer (ITS) sequencing was done for identification. The Zygomycetes spp. was identified as Rhizopus microsporus. Alignment was made with the reference strain of international collection (Figure 4).

After confirming gastric mucormycosis, Amphotericin-B (AmB) $200 \mathrm{mg}$ in $500 \mathrm{ml}$ 5\% Dextrose was started slowly intravenously

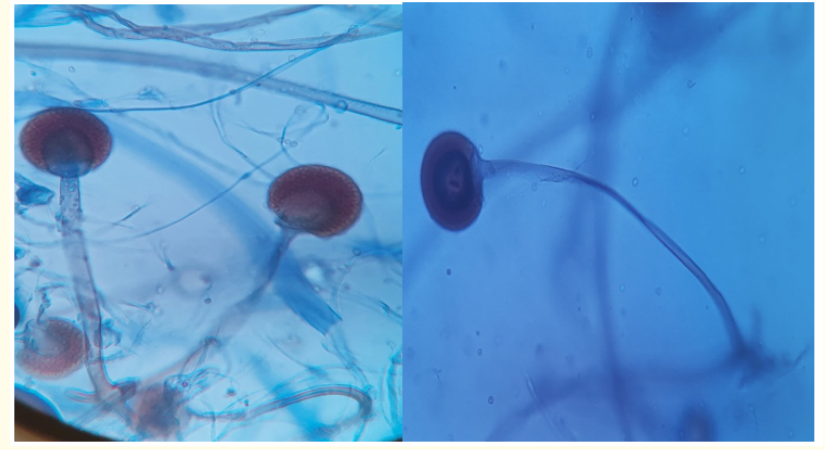

Figure 3: LPCB mount shows broad based hyphae with nodal rhizoids, short, brown sporangiophores and sporangia containing sporangiospores.

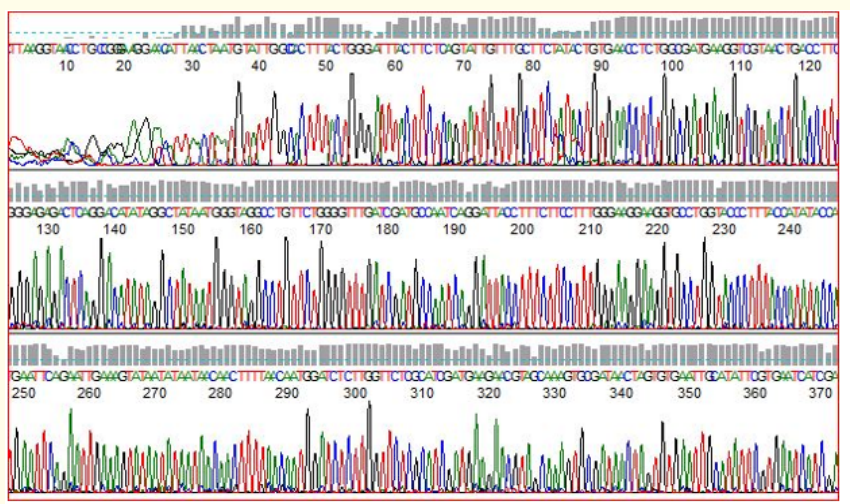

Figure 4: Part of Internal Transcribed Spacer Sequencing of Rhizopus microspores.

over a period of 5 - 6 hours. However, due to deranged renal function (elevated serum creatinine, $2.51 \mathrm{mg} / \mathrm{dl}$ ) and hypokalemia of $2.7 \mathrm{mmol} /$ after 4 days of $\mathrm{AmB}$, it was stopped. Later, he was started on Posaconazole (Picasa, Intas Pharmaceuticles) $200 \mathrm{mg}$ three times daily orally for 3 weeks. Patient became afebrile. After thirteen days he was discharged in stable condition. Repeat UGIE was done after 4 weeks. Ulcer was healing but some raw area and necrosis was persisting, perforation healed completely. Posaconazole was further given for 2 weeks in same doses. Repeat UGIE 
after completion of next 2 week therapy showed complete healing. Patient is still under follow up and he is doing well.

\section{Discussion and Conclusion}

GIM are quite rare over the globe; however, there are few reported cases in the literature over last two decades. Gastrointestinal mucosal involvement could be the sole manifestations of mucormycosis that accounts for approximately 7\% all reported cases [12]. Amongst the Rhizopus spp., Rhizopus arrhizus is the most common species causing mucormycosis across the globe [3]. Here, we are presenting a case of gastric mucormycosis by Rhizopus microsporus.

The gastric mucormycosis may be the result of ingestion or direct inoculation of sporangiospores onto disrupted mucosa in immunocompromised individuals [11]. The symptoms of GIM are varied and depend on the affected site. Abdominal pain and distention associated with nausea, vomiting, fever, diarrhea, hematochezia are common symptoms [3,9]. Non-specific symptomatology and delayed antepartum diagnosis are the cause of very high mortality (upto 85\%) in GIM [12].

The prevalence rate of mucormycosis amongst patients with uncontrolled diabetes is $36 \%$ [13]. Three major case series from India reported diabetes as a risk factor over $50 \%$ cases with mucormycosis [14]. Although rhinocerebral mucormycosis is most commonly associated with DM, cases of GIM have also been found associated with it [12]. Uncontrolled DM has been one of the major risk factors for GIM (17\% to $88 \%$ ) [3].

Commonly used antifungal medications are lipid formulation of amphotericin B (AmpB) (45\% to 52\%) and posaconazole (45\%) in hospitalized patients with mucormycosis [3]. However, a combination of antifungal with surgical management with either debridement or gastrectomy of mucormycosis lesions may be required in a significant number of cases specifically in those with severe disease and multiple comorbidities [7,11]. In our case, he was started on (AmpB); however due to derange renal function, Posaconazole was started orally. Patient got improved with Posaconazole therapy (Figure 5).

High index of clinical suspicion in patient with uncontrolled DM coming with non-specific symptoms like fever, vomiting, hematemesis, melena can help in diagnosis. Early diagnosis can be made by histopathological examination of the biopsy sample, $\mathrm{KOH}$

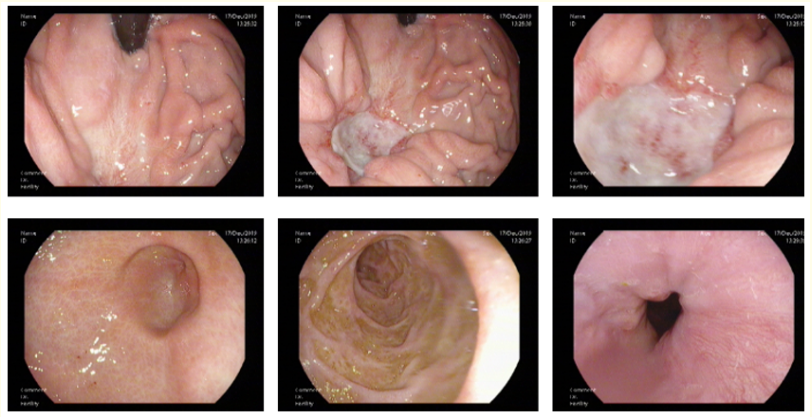

Figure 5: Healed ulcer after antifungal treatment.

mount of the fluid aspirated or tissue or by endoscopy. Definitive diagnosis is by microbiological culture and identification of the causative organism on the basis of colony morphology. Species level identification can be done by molecular sequencing method. Molecular identification up to species level helps in formulating empiric treatment in the geographical area and for the management of patients reducing the mortality rate.

\section{Conflict of Interest}

There was no conflict of interest to declare.

\section{Funding}

There was no source of funding.

\section{Bibliography}

1. Hibbett DS., et al. "A higher-level phylogenetic classification of the Fungi". Mycological Research 111 (2007): 509-547.

2. Dikshya Sharma., et al. "Case of early-disseminated Rhizopus microsporus var. microsporus mucormycosis in a renal transplant patient". International Medical Case Reports Journal 9 (2016): 139-143.

3. Prakash H and Chakrabarti A. "Global Epidemiology of Mucormycosis". Journal of Fungi (Basel) 5.1 (2019): 26.

4. A Serris., et al. "Disease entities in mucormycosis". Journal of Fungi 5.1 (2019): 23.

5. Salwa O Mekki., et al. "Pulmonary Mucormycosis: A Case Report of a Rare Infection with Potential Diagnostic Problems". Case Reports in Pathology 2020 (2020): 5845394. 
6. Kaur H., et al. "Gastrointestinal mucormycosis in apparently immunocompetent hosts-A review". Mycoses 61 (2018): 898908.

7. Kontoyiannis DP and Lewis RE. "Invasive zygomycosis: update on pathogenesis, clinical manifestations, and management". Infectious Disease Clinics of North America 20 (2006): 581-607.

8. Epifanio Silvino do Monte Junior., et al. "Rare and Fatal Gastrointestinal Mucormycosis (Zygomycosis) in a COVID-19 Patient: A Case Report”. Clinical Endoscopy 53.6 (2020): 746-749.

9. Maraví-Poma E., et al. "Outbreak of gastric mucormycosis associated with the use of wooden tongue depressors in critically ill patients". Intensive Care Medicine 30.4 (2004): 724-728.

10. Shiva Prasad BN., et al. "Primary gastrointestinal mucormycosis in an immunocompetent person". Journal of Postgraduate Medicine 54 (2008): 211-213.

11. Spellberg B. "Gastrointestinal mucormycosis: an evolving disease". Gastroenterology and Hepatology (N Y) 8 (2012): 140142.

12. Maureen M Roden., et al. "Epidemiology and outcome of zygomycosis: a review of 929 reported cases". Clinical Infectious Diseases 41.5 (2005): 634-653.

13. A Vera., et al. "Invasive gastrointestinal zygomycosis in a liver transplant recipient". Transplantation 73.1 (2002): 145-147.

14. Chakrabarti A., et al. "The rising trend of invasive zygomycosis in patients with uncontrolled diabetes mellitus". Medical Mycology 44.4 (2006): 335-342.

\section{Volume 4 Issue 6 June 2021}

(C) All rights are reserved by Gitali Bhagawati., et al. 